\title{
Aprendizaje-servicio: una experiencia rural de promoción de la salud con varones adultos en Tucumán (Argentina)
}

\section{Analía Graciela Soria}

Docente de la Facultad de Bioquímica

Química y Farmacia. Universidad

Nacional de Tucumán, Argentina.

draanaliasoria@arnet.com.ar

\section{Rosa Silvina Guber}

Docente de la Facultad de Bioquímica, Química y Farmacia. Universidad Nacional de Tucumán.

rsguber@hotmail.com

\section{Liliana Mónica Tefaha}

Docente de la Facultad de Medicina. Universidad Nacional de Tucumán.

Itefaha@gmail.com

\section{Claudio Romero}

Docente de la Facultad de Medicina Universidad Nacional de Tucumán. klaudioromero@yahoo.com.ar
Integración de la docencia y la extensión /

Intervenciones

RECEPCIÓN: 24/06/16

ACEPTACIÓN FINAL: 10/10/16

\section{Resumen}

El objetivo de esta experiencia fue aumentar la demanda en salud en varones adultos. Para ello se capacitó a estudiantes de Facultad de Medicina, que realizaban su pasantía rural en Graneros, y de Bioquímica de la Universidad Nacional de Tucumán para la aplicación de un programa local de salud destinado a la promoción, detección de factores de riesgo y enfermedades prevalentes en varones adultos. Participaron 66 estudiantes de Medicina y 23 de Bioquímica. Las estrategias más usadas fueron: difusión radial, carteles en lugares de mayor concurrencia, visita domiciliaria con invitación a participar. Los estudiantes evaluaron la actividad como una experiencia positiva. El $70 \%$ de ellos tuvo muy buen desempeño. De los 678 varones invitados, el $40 \%$ concurrió para la atención y se lo incluyó en un programa de seguimiento en centro de Atención Primaria de la Salud. El trabajo de modo sistemático desde el grado favorece en los estudiantes el desarrollo de habilidades y modifica su percepción de las necesidades reales de salud en poblaciones deprimidas.

\section{Palabras-clave}

- Aprendizaje y servicio

- Patologías prevalentes

- Factores de riesgo

- Responsabilidad

- Multidisciplina

\section{Resumo}

O objetivo desta experiência foi aumentar a demanda da saúde em homens adultos. Para isso se capacitou estudantes da Faculdade de Medicina -que realizavam o seu estágio rural em Graneros-e de Bioquímica da Universidad Nacional de Tucumán, para a implementação de um programa de saúde local para a promoção, detecção de fatores de risco e doenças prevalentes em homens adultos. Participaram 66 estudantes de medicina e 23 de bioquímica. As estratégias mais utilizadas foram: radiodifusão, cartazes em locais de maior afluência, visita domiciliar com convite para participar. Os estudantes avaliaram a atividade como uma experiência positiva. $70 \%$ deles tiveram desempenho muito bom. Dos 678 homens convidados, 40\% compareceu para cuidados e foi incluído em um programa de monitoramento no centro de Atenção Primária à Saúde. O trabalho sistemático a partir da graduação favorece os alunos no desenvolvimento de habilidades e modifica sua percepção das necessidades reais de saúde em populações vulneráveis.

\section{Palavras-chave}

- Aprendizagem e serviço

- Patologias prevalentes

- Fatores de risco

- Responsabilidade

- Multidisciplinar

\section{Para citación de este artículo}

Soria, G.; Guber, R. Tefaha, L. y Romero, C. (2016).

Aprendizaje-servicio: una experiencia rural de promoción de la salud con varones adultos en Tucumán (Argentina). En Revista +E versión digital, (6), pp. 278-285. Santa Fe, Argentina: Ediciones UNL. 


\section{Introducción}

El aprendizaje-servicio (AS) ha crecido muy rápidamente en Argentina y puede ser definido como

"una actividad o programa de servicio solidario protagonizado por los estudiantes, orientado a atender eficazmente necesidades de una comunidad, y planificada en forma integrada con los contenidos curriculares en función de optimizar los aprendizajes". (Tapia, 2008:22)

Los estudiantes tienen la oportunidad de aplicar los conocimientos y competencias del perfil profesional para contribuir a la comprensión y resolución de problemas cuyo objetivo final es mejorar las condiciones de vida de una comunidad. Una forma en que los estudiantes adquieren las habilidades y competencias es a través de un modelo conceptualizado como Aprendizaje Basado en Competencias, definido por la Organización para la Cooperación y el Desarrollo Económico como la combinación de habilidades prácticas, conocimientos, motivación, valores éticos, actitudes, emociones y otros componentes sociales que se movilizan conjuntamente para lograr una acción eficaz (Hersh, et al., 1999). Existen numerosos antecedentes de experiencias en AS a nivel mundial evaluadas como satisfactorias para los profesores y los estudiantes de distintas carreras y países, como los de magisterio en España (Gervilla Zapata y otros, 2011), médico en Japón (Okayama y Kajii, 2011) y en Venezuela, entre otros. En la Argentina, la Universidad Nacional de Río Cuarto, a través de la OCS n³22/09 ha implementado módulos de Prácticas Sociocomunitarias en todos los currículos de grado de las carreras que allí se dictan. Asimismo, la Universidad de Buenos Aires, mediante la Resolución $n^{\circ}$ 520/10 del Consejo Superior, ha puesto en funcionamiento un Programa Universitario de Prácticas Educativas Solidarias, donde los estudiantes de todas las carreras de grado deben cumplimentar 42 horas de Prácticas. En la Universidad Nacional de Tucumán (UNT), la carrera de Médico tiene incorporada en su currículo una pasantía rural obligatoria de seis meses durante el séptimo año desde 1989. Durante la misma los estudiantes deben residir en uno de los 28 Centros de Atención Primaria (CAPS) seleccionados por la Facultad ubicados en el interior de la provincia de Tucumán, Salta, Jujuy y Santiago del Estero. Existen convenios con el sistema de salud por los cuales la Facultad se compromete a aportar el acervo bibliográfico, material y equipamiento necesario para la permanencia de los estudiantes, y el sistema de salud se compromete a brindar el espacio físico y la comida. Deben cumplir sus actividades de lunes a viernes y realizar además una guardia semanal. Estas sedes cuentan con un médico del servicio que es capacitado por la Facultad para cumplir las funciones de instructor docente y guiar y supervisar a los pasantes. Igualmente, tienen como actividad obligatoria el diagnóstico de salud del área de responsabilidad y, sobre la base de las necesidades detectadas, la realización de programas locales de salud y la participación en programas nacionales, provinciales y locales existentes. En este escenario, los estudiantes tienen diversas oportunidades de efectuar prácticas solidarias donde se integran los saberes científico-técnicos adquiridos en la academia con las realidades sociales que les toca vivir. Como tienen una formación epidemiológica previa, pueden hacer diagnósticos de salud de la comunidad y seleccionar las intervenciones adecuadas. Los alumnos de la carrera de Bioquímica no contemplan en su currículo este tipo de prácticas dado que su incorporación fue voluntaria (extracurricular). En Graneros, localidad situada en la zona este de la provincia de Tucumán, funciona uno de estas sedes donde los pasantes toman contacto con la realidad social.

Al finalizar la pasantía como requisito final para obtener su título de Médico, rinden un examen grupal y otro individual donde deben presentar las acciones desarrolladas y un trabajo de investigación epidemiológica. A partir de estas presentaciones, se observó que los programas están fundamentalmente orientados a la salud del niño y de la madre. El trabajo final de carrera debe tener como motivo de investigación alguno de los problemas de salud detectados. Estas tesinas se encuentran en la biblioteca de la Facultad de Medicina y están agrupadas por problemas de salud. En la actualidad existen más de 2000 trabajos realizados de los 
cuales aproximadamente un $20 \%$ se refiere a la problemática de las enfermedades crónicas prevalentes. En ellos se ha demostrado que las consultas para detección o control de enfermedades son muy escasas y que los varones adultos (VA) casi nunca concurren a los CAPS por coincidir los horarios de atención de los servicios con sus horas de trabajo. Estos datos se corresponden con los de la encuesta de Utilización y Gastos en Servicios de Salud (2006) de la Dirección de Estadísticas e Información del Ministerio de Salud, realizada en el área Metropolitana, donde se destaca que la consulta por prevención es más mencionada por mujeres, por los que tienen alguna cobertura en salud y por la población de mayores ingresos y niveles educativos más altos. Esta problemática de baja cobertura y escasa demanda en salud de este grupo etario se acrecienta por la falta de adherencia a las medidas de promoción, detección y tratamiento precoz (Dryden et al., 2012). A ello se agrega que estas patologías pueden permanecer mucho tiempo asintomáticas y por lo tanto no motivan la consulta, como por ejemplo sucede con la hipertensión arterial, la diabetes, el síndrome metabólico y las complicaciones que de ellas derivan que, junto con los tumores, como el cáncer de próstata, constituyen para el VA las primeras causas de morbimortalidad tanto en nuestro país como en el mundo (INDEC, 2012). A su vez tienen la contrapartida que pueden ser prevenidas o modificadas en su evolución a través de acciones de promoción o medidas de prevención de bajo costo y alta eficacia.

A partir de esta problemática se decidió realizar un programa de salud local piloto destinado a aumentar la demanda de salud y la cobertura de los VA. Se seleccionó la pasantía de Graneros por ser una de las poblaciones más deprimidas, con un alto porcentaje de familias con Necesidades Básicas Insatisfechas. De esta manera se buscó formar desde el grado profesionales médicos y bioquímicos con enfoque de Atención Primaria de la Salud, conocedores y comprometidos con las características y necesidades de la comunidad donde se encuentran insertos, con la finalidad de llevar a cabo acciones efectivas de promoción de salud y prevención de enfermedades prevalentes del VA.

\section{Objetivos específicos}

Es necesario que los estudiantes de Medicina y Bioquímica logren: - Desarrollar habilidades comunicacionales durante la entrevista clínica para la detección de factores de riesgo modificables y educación en salud.

- Adquirir habilidades y destrezas en la realización del examen físico dirigido a la búsqueda de patologías prevalentes. Adquirir competencias en habilidades de comunicación y participación social y comunitaria.
- Fortalecer su formación en el conocimiento clínico-patológico y bioquímico, de las enfermedades prevalentes en VA facilitando la realización de procesos de integración de los mecanismos patogénicos de enfermedades con las determinaciones bioquímicas.

- Adquirir las habilidades y destrezas en las determinaciones de rutina en el laboratorio y sistematización del trabajo.

- Fortalecer la capacidad reflexiva y juicio crítico.

- Llevar a cabo procesos de autoevaluación permanente como mecanismos de retroalimentación en los distintos escenarios de enseñanza-aprendizaje, durante las prácticas a través de reuniones periódicas para ajuste de procedimientos y discusión de casos, con apoyo científico-técnico de las cátedras pertinentes. - Desarrollar habilidades para el trabajo en equipos multidisciplinarios, orientados por docentes universitarios y personal de salud de los CAPS.

- Adquirir capacidad para efectuar acciones integradas de gestión y participación comunitaria e intersectorial a través de las redes de salud demostrando actitudes éticas.

A nivel de la comunidad:

- Aumentar la cobertura y demanda en salud en VA.

- Identificar factores de riesgo modificables (prevención primaria).

- Detectar y controlar patologías prevalentes (prevención secundaria).

- Fomentar la adherencia a los tratamientos.

\section{Material y métodos}

Las acciones generadas por el programa permitieron obtener datos sobre la población de VA y sobre las capacidades adquiridas por los estudiantes, así como acerca del grado de satisfacción con las actividades realizadas. A partir de estos datos se realizó un estudio descriptivo de corte transversal y de carácter exploratorio que comprendió los años 2008 a 2012 en la población objetivo: VA de Graneros, provincia de Tucumán, Argentina, donde funciona una de las sedes de pasantía rural de la Facultad de Medicina. Como dura seis meses, durante los cinco años que abarcó el estudio se incorporaron 10 cohortes de estudiantes. En cada ingreso a la pasantía se efectuó un taller de capacitación en el que, además de los docentes, participaron los estudiantes que egresaban con la finalidad de que los mismos realizaran una autoevaluación de las acciones ejecutadas, transmitieran a los ingresantes las experiencias y vivencias adquiridas e hicieran sugerencias tendientes a mejorar las acciones. Participaron 66 estudiantes de Medicina y 23 de Bioquímica. Cada cohorte fue capacitada mediante una Jornada Taller en recursos usados en promoción de salud y prevención 


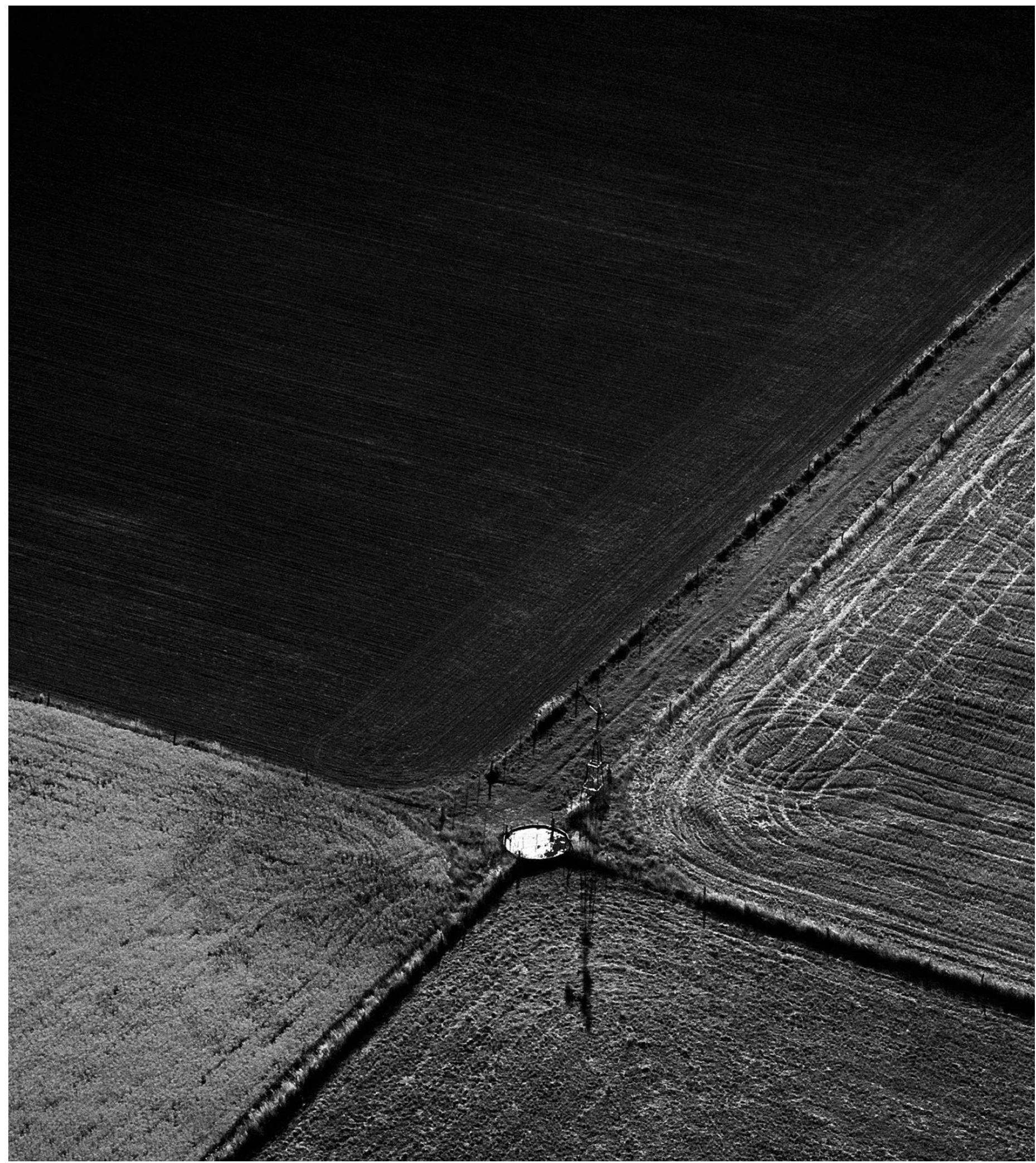

(c) Oscar Dechiara 
de enfermedades; relación médico-paciente-comunidad; uso de estrategias durante la entrevista clínica para detección de Factores de Riesgo Modificables; recursos de educación médica para la adopción de medidas preventivas y reconocimiento de síntomas de alarma y estrategias para la preparación y coordinaciones de talleres de educación en salud dirigidos a la población objetivo. Las estrategias metodológicas fueron: discusión en pequeños grupos, rol playing, mostración-demostración, realización de prácticas específicas como toma de presión arterial, glucemia capilar, peso, talla, cálculo del índice de masa corporal. Los estudiantes de Medicina desarrollaron, con supervisión docente y de manera sistemática, las siguientes actividades de trabajo en terreno: difusión semanal en micros de educación para la salud en la radio local; programas y talleres con participación comunitaria orientados a prevención y detección precoz sobre Factores de Riesgo Modificables; participación en la elaboración de encuestas para la recolección de datos epidemiológicos, clínicos y de laboratorio; visita domiciliaria para invitar a los VA a concurrir al centro de atención primaria en fecha y hora previamente establecidas. Durante esa visita debían explicar en qué consistía el programa y cuál era el objetivo del mismo, aprovechando la ocasión para realizar promoción de la salud.

Los varones que concurrieron al centro de atención firmaron el consentimiento informado para la realización de los estudios en forma voluntaria y gratuita. Los estudiantes efectuaron entrevista clínica bajo supervisión docente orientada a la detección de Factores de Riesgo Modificables, examen físico con control de presión arterial, peso, estatura y cálculo del Índice de Masa Corporal. Los estudiantes de Bioquímica, supervisados por los docentes, realizaron extracciones de sangre venosa. En los sueros, se efectuaron las siguientes determinaciones: antígeno prostático específico, colesterol total y glucemia. Se llevaron a cabo reuniones entre médicos, bioquímicos y estudiantes participantes para la evaluación de historias clínicas con el fin de hacer devolución a cada uno de los VA que concurrieron a la consulta. En los casos en que se detectaron hipertensión arterial, diabetes u otra patología crónica, se los referenció al Centro de Salud para su control y seguimiento. El desempeño de los alumnos fue evaluado por observación directa del docente mediante listas de cotejo donde se calificó: habilidad comunicacional en la relación médicopaciente, capacidad para orientar la anamnesis a la búsqueda de factores de riesgo y de efectuar el examen físico, habilidad para brindar consejería y capacidad de organizar y llevar a cabo los talleres comunitarios.

El nivel de satisfacción y compromiso de los estudiantes respecto de las actividades realizadas se evaluó mediante encuestas anónimas seis meses después de haber participado de la experiencia de aprendizaje-servicio (final de su pasantía).

\section{Resultados}

\subsection{Resultados en estudiantes}

De los 10 talleres de capacitación se obtuvieron los siguientes resultados: todos los estudiantes participaron activamente en las actividades del AS. Generaron 10 trípticos con material informativo que fueron evaluados por los docentes como muy buenos a excelentes. Este material fue empleado en los 14 talleres con participación comunitaria. En estos talleres, la comunidad realizó sugerencias para optimizar la difusión, como hacer carteles y colocarlos en sitios estratégicos (almacén, farmacia, verdulería) ofreciendo controles gratuitos. El $70 \%, 25 \%$ y el $5 \%$ de los alumnos fueron calificados por los docentes con desempeño muy bueno, bueno, y no satisfactorio, respectivamente. Las mayores dificultades se observaron en la capacidad de establecer una comunicación adecuada durante la entrevista clínica, especialmente en cuanto a brindar información y consejería con lenguaje adecuado y al uso correcto de instrumentos de medición (tensiómetro, monitor de glucemia capilar).

\subsection{Resultados en la comunidad}

Fueron visitados en su domicilio 678 VA. El $40 \%$ de los mismos $(n=271)$ concurrió a los CAPS para su atención y fue incluido en el programa. De ellos, el 38,4 \% regresó dentro del año, en forma espontánea, para un nuevo control periódico de salud. A partir de los datos de la encuesta y de la entrevista clínica se pudo caracterizar la población objetivo. Las Figuras 1, 2 y 3 muestran las características sociodemográficas. El grupo de varones estudiado tiene bajo nivel de educación; el 57,9 \% estaban casados o vivían con su pareja; el 53,5 \% se trataba de empleados remunerados. Las Figuras 4, 5, 6, 7 y 8 expresan los factores de riesgo modificables entre los cuales podemos destacar hipercolesterolemia (51,7 \%); tabaquismo (60,5\%); consumo de alcohol (54,2\%), de los cuales el $42,9 \%$ consume más de un litro por día; la ingesta de carnes rojas con grasas (86,3 \%); Índice de Masa Corporal (sobrepeso 42,8 \% y obesos 25,1\%). En las Figuras 9, 10, 11 y 12 se muestran los problemas de salud encontrados. El agrandamiento de la próstata detectado por ecografía fue el más frecuente, seguido por la hipertensión arterial, diabetes y cáncer de próstata.

\subsection{Resultados del nivel de satisfacción de los estudiantes} De las encuestas realizadas a los estudiantes de ambas carreras, el 100 \% está "muy de acuerdo" con la realización de estas prácticas, y los de Bioquímica enfatizan la importancia de desarrollar estas actividades para comprender mejor las incumbencias profesionales y poder así actuar en equipos de salud multidisciplinarios para resolver problemas de la comunidad. Algunas de las expresiones frecuentes de los estudiantes fueron: 
Fig. 1. Educación



Ninguna, Primaria Completa o Primaria Incompleta

Estudios Secundarios

Estudios Superiores

Fig. 5. Consumo de tabaco



$\mathrm{Si}$

No
Fig. 2. Estado civil

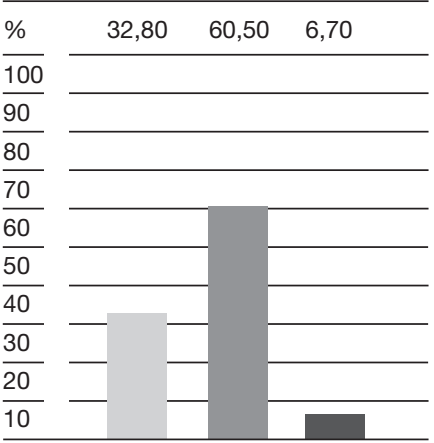

$\square$ Soltero

$\square$ Casado o en pareja

$\square$ Viudo
Fig. 3. Ocupación

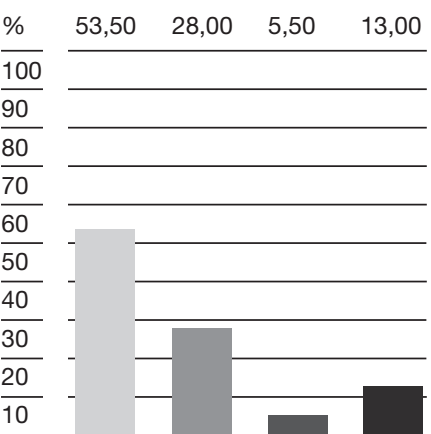

$\square$ Empleado

$\square$ Jubilado

- Desocupado

Trabajadores Golondrinas

Fig. 6. Consumo de alcohol

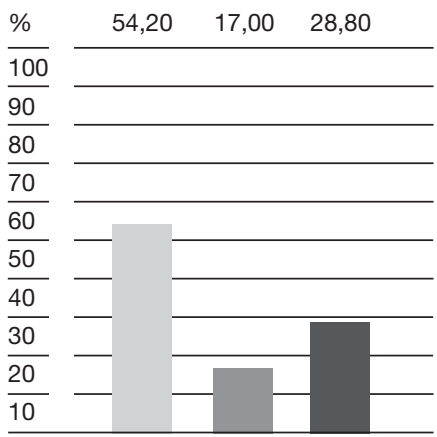

$\square \mathrm{Si}$

$\square$ No

$\square$ No contesta

Fig. 10. Diabetes Mellitus

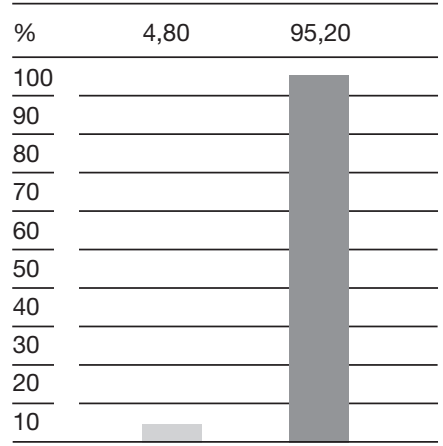

$\square \mathrm{Si}$

$\square$ No
Fig. 7. Consumo de carnes rojas

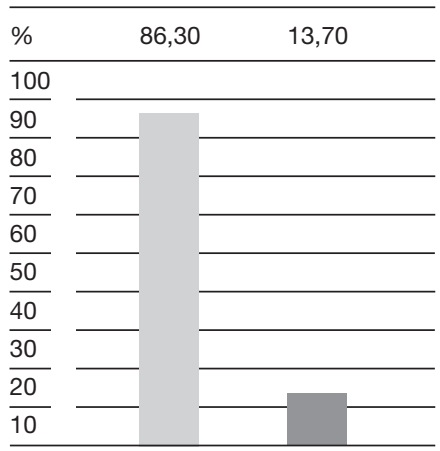

$\square \mathrm{Si}$

$\square \quad$ No

Fig. 11. Agrandamiento de la próstata

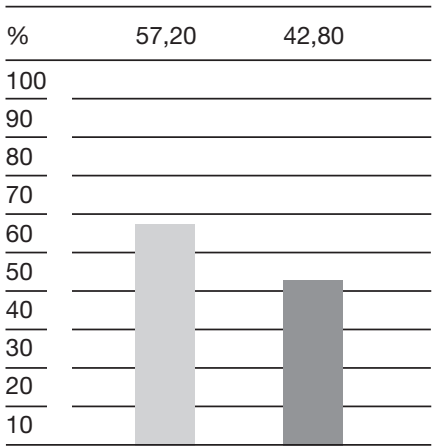

$\square \mathrm{Si}$

$\square$ No
Fig. 4. Colesterol

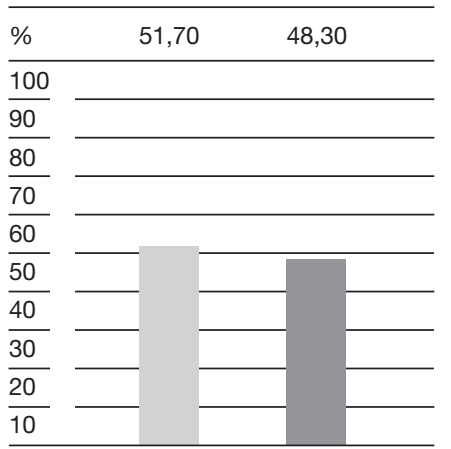

$\square>200 \mathrm{mg} / \mathrm{dl}$

$\square<200 \mathrm{mg} / \mathrm{dl}$

Fig. 8. Índice de masa corporal

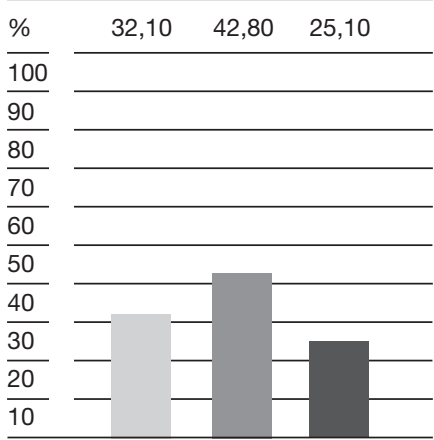

$\square<25 \mathrm{Kg} / \mathrm{m} 2$

$25-30 \mathrm{Kg} / \mathrm{m} 2$

$>30 \mathrm{Kg} / \mathrm{m} 2$




"Nos mostró un área poco conocida en la enseñanza de la Medicina"; "nos hizo ver la poca habilidad que tenemos en la relación médico-paciente"; "nos capacitó de manera sistemática en la utilización de metodologías para el abordaje comunitario"; "descubrí que con un grupo multidisciplinario, médicos, bioquímicos y estudiantes, participando cada uno, desde su rol, puede ofrecer un gran servicio a la comunidad, a gente que no tiene las mismas posibilidades que el resto de la población pero que sí tienen el mismo derecho a participar de un buen sistema de salud".

\section{Discusión y conclusión}

Este tipo de actividades persigue un doble objetivo, por un lado, la educación de la comunidad para promoción en salud y detección precoz de enfermedades prevalentes, y por otro lado, la adquisición de competencias de estudiantes en esta área. La Facultad de Medicina de la UNT, al tener incorporada una pasantía rural en su plan de estudio como requisito final para obtener el título de Médico, facilita la implementación y continuidad de programas locales de salud orientados a las problemáticas de salud de la población de referencia o área de responsabilidad del CAPS. Las enfermedades cardiovasculares, cáncer, enfermedades respiratorias crónicas y diabetes constituyen una problemática de salud no solo a nivel local sino a nivel global, ya que son responsables de alrededor de $60 \%$ de muertes en el mundo, $80 \%$ de ellas ocurre en países de ingresos bajos y medios (Mathers et al., 2004). En nuestro país, como consecuencia del cambio demográfico, se incrementa la prevalencia de la población añosa, por lo que habrá que implementar estrategias que disminuyan la mortalidad y el costo de la discapacidad de las enfermedades no transmisibles. Es por ello importante identificar los factores y poblaciones en riesgo para aplicar medidas preventivas. En los VA de Graneros se observó una alta frecuencia de factores de riesgo modificables para enfermedades cardiovasculares, por lo que los datos aportados refuerzan la necesidad de implementación de programas de prevención de estas patologías. En la población estudiada se pudo observar un porcentaje mayor de sobrepeso y obesidad (43\% y $25 \%$ ) que los datos registrados en la Encuesta nacional de factores de riesgo 2009 (35,4 y $18 \%$ ). La presencia de hipercolesterolemia también fue mayor (52 \% vs. 29,1\%) al igual que el consumo de tabaco (60 \% vs. $30,1 \%)$. Sin embargo la prevalencia de diabetes $(4,8 \%$ vs. 9,6 \%) y de hipertensión arterial (19,6 vs. 34,8 \%) fue mucho menor. Si bien hay un alto porcentaje de VA con agrandamiento de próstata (57\%), cuando se lo compara con publicaciones internacionales que encuentran un $13,5 \%$, los casos detectados con cáncer de próstata $(2 \%)$ son menores que los publicados por otros autores, quienes hallan una prevalencia del 7,8 \% (Issa, et al., 2006). El proyecto tuvo impacto positivo tanto en la comunidad

como en docentes y estudiantes. En la comunidad generó alternativas para el control de salud al que tiene derecho todo hombre, sobre todo si se considera que el diagnóstico precoz de patologías prevalentes, su tratamiento oportuno y la educación sobre factores de riesgo modificables aumentan las probabilidades de curación y la posibilidad de reducir las altas tasas de mortalidad de las enfermedades no transmisibles. Se logró una mayor autoconciencia acerca de la importancia de participar en los programas para detección de enfermedades en general (como concurrir a un control médico anual aun cuando no se tengan síntomas) y, secundariamente, la importancia de participar en programas de detección precoz para cáncer de próstata, conocer factores de riesgo y síntomas de alarma.

A partir de estas acciones, se pudo observar que los pacientes en los cuales se detectó hipertensión arterial y/o diabetes continuaron yendo en forma periódica al CAPS y fueron incluidos en el Programa REMEDIAR. ${ }^{1}$ Un 38 \% concurrió en forma espontánea dentro del año de su primera visita para realizarse un nuevo control de salud, y además fue el grupo que continuó participando en los talleres de educación para la salud realizados por los estudiantes. En esta segunda visita, un $15 \%$ manifestó haber realizado algún tipo de actividad física. Un $5 \%$ dijo haber dejado de fumar. Solo 5 pacientes dijeron haber modificado su dieta. Las causas más frecuentes relacionadas con la ausencia de adherencia al ejercicio y dieta fueron "la falta de tiempo y razones económicas". En cuanto a los docentes, permitió que cambien las clases magistrales por la aplicación de conocimientos en el campo de acción y que los estudiantes desarrollen competencias en la relación médicopaciente-comunidad. La motivación y el interés de los mismos aumentaron durante el desarrollo de la práctica, lo cual favoreció las habilidades comunicacionales, modificó la percepción sobre las necesidades reales de salud en poblaciones deprimidas, excluidas, e hizo posible que adquirieran competencias formativas y en gestión para la formación de redes de salud donde la multidisciplina e intersectorialidad tuvieron un rol preponderante.

Los estudiantes de Medicina, al residir en la localidad, forman parte de la misma y participan en todos los eventos tanto sociales como culturales. Resulta interesante el cambio de actitud gradual que se va dando en los mismos a medida que transcurre la pasantía. Si bien durante la carrera realizan de primero a quinto año un eje transversal de salud pública, donde hacen prácticas en terreno y trabajos de campo, es recién durante la pasantía rural cuando asimilan realmente cómo vive, cómo se enferma y cómo muere la gente. En general, tienen gran resistencia a realizar esta parte

1) El programa Remediar contempla la provisión gratuita de medicamentos ambulatorios a los ciudadanos que es- tán bajo línea de pobreza o no tienen cobertura de obra social. 
de su trayecto curricular, pero con el transcurso de los días estas barreras se van venciendo, tal como lo demuestran los testimonios expresados anteriormente. Esto favorece su responsabilidad como futuros profesionales de la salud y afianza las relaciones interpersonales entre médico y bioquímico para su trabajo conjunto, lo que los obliga a interrelacionarse y coordinar acciones conjuntas, fortalecer las actitudes de cooperación, de competencias leales, de motivación de los compañeros para lograr los objetivos evitando los conflictos interpersonales. Así se enriqueció la diversidad y complejidad del planteamiento de ideas y enfoques para perfeccionar cada vez más la atención eficiente del paciente. En los diversos talleres planificados en cada etapa del Proyecto, los estudiantes generaron ideas, hipótesis, desarrollaron actitudes críticas, identificaron alternativas en las distintas situaciones planteadas. En resumen, esta práctica favorece en los estudiantes el compromiso, conciencia social, solidaridad y responsabilidad profesional personal y colectiva. A partir de esta experiencia, sería necesario incorporar en forma obligatoria el AS, como ya ocurre en otros países y otras universidades de Argentina, como actividad previa a la graduación de los estudiantes, lo que les permitiría reconocer en forma real escenarios posibles de su futuro desempeño profesional.

\section{Bibliografía}

Gervilla Zapata, M. y otros (2011). Experiencia de Aprendizaje Servicio por Estudiantes de Magisterio de la Universidad de Granada. V Congreso Universidad y Cooperación. Universidad de Granada. Recuperado de: http://www.quintocongresocud.es/pdf/806.pdf (10/09/2012).

Hersh, L.; Simone, D.; Moser, U. y Konstant, J. (1999). Proyectos sobre competencias en la OCDE. París: OCDE.

INDEC (2012). Mortalidad. Recuperado de: http://www.indec.mecon.ar/principal. asp?id_tema $=66(11 / 09 / 2012)$

Issa, M. M. et al. (2006). Assessment of the Diagnosed Prevalence of Diseases in Men 50 Years of Age or Older. Am J Manag Care, 12, S83-S89.

Mathers, C. D. et al. (2004). Deaths and Disease Burden by Cause: Global Burden of Disease Estimates for 2001 by World Bank Country Groups. Disease Control Priorities Project Working. Recuperado de: http://www.dcp2.org/file/33/wp18.pdf (10/09/2012)

Okayama, M.; Kajii, E. (2011). BMC Medical Education 11:11-19. Recuperado de: http://www.biomedcentral.com/1472-6920/11/19 (13/09/2012).

Tapia, M. N. (2008). Aprendizaje y servicio solidario en la misión de la Educación Superior. Recuperado de: http://www.me.gov.ar/edusol/archivos/2008_as_edu_ sup.pdf (10/09/2012).

\section{G6}

en cuanto a los docentes, permitió que cambien las clases magistrales por la aplicación de conocimientos en el campo de acción y que los estudiantes desarrollen competencias en la relación médicopaciente-comunidad 